\title{
Economic evaluation of early intervention services
}

\author{
PAUL MCCRONE and MARTIN KNAPP
}

\begin{abstract}
Summary Early intervention services have been introduced in a number of countries, but the evidence base to support them is limited. In particular there are very few economic evaluations, which are crucial if decision-makers are to have a better understanding of how scarce
\end{abstract} resources can be used appropriately. This paper discusses the different approaches used in economic evaluations and shows how these differ in the way in which outcomes are measured. The most useful forms of evaluation are cost-effectiveness and cost-utility analysis. We describe how the results of evaluations can be interpreted using incremental costeffectiveness ratios and acceptability curves. Finally, the paper summarises some key evidence to date on early intervention services and economic evaluations currently being undertaken.

Declaration of interest None.

The early intervention approach has its roots in the early psychosis initiatives in the UK, Australia, Canada, New Zealand, Scandinavia, Germany and the Netherlands from the 1990s. The first early intervention service in the UK started in Birmingham in 1990. In England there is a national policy requirement that such services be established (Department of Health, 2001), with the expectation that early intervention teams should each cover a population of around 1 million people. Clearly there is a cost associated with such provision and resources employed by these teams could be used in other areas of mental healthcare (for example providing greater capacity for assertive outreach teams or more acute beds), or for other conditions (cancer, asthma, etc.) or for non-health purposes (education, defence, etc.). The fact that healthcare resources are limited in their supply, coupled with the recognition that the demand, or need, for healthcare is considerable, implies that we should gauge the level of benefit or outcome that can be achieved from money spent on a particular healthcare intervention and compare this with similar data for other interventions in the same area and interventions elsewhere. Economic evaluations, in theory, perform this role. Costs represent care inputs, which should be determined by the needs of patients, and hopefully desirable outcomes can be achieved from those inputs.

\section{THE COST OF EARLY INTERVENTIONS}

One of the key aims of early intervention services is to reduce the duration of untreated psychosis (DUP). This is important as prolonged DUP can obviously be distressing to patients and those around them and there is also evidence that it results in poorer outcomes once treatment begins (Marshall et al, 2006). Prolonged DUP is likely to have a cost impact. While untreated, people with early stage psychosis may find it difficult to remain in employment or education. In addition they may have disproportionately high contact with general health services and the criminal justice system. Once treatment begins, it may need to be more prolonged than if the DUP was shorter, and poorer outcomes will likely necessitate continued use of psychiatric services to a greater degree than if there was a shorter DUP.

It is important to adopt a comprehensive perspective when considering the costs associated with early intervention services. Clearly it is crucial to measure the costs of the team itself, but an evaluation should also measure the cost of in-patient care, other mental health services, general healthcare, care provided by social services, inputs from education agencies, and contacts with the criminal justice system. Furthermore, family members or friends will also provide care for many patients. This will usually be unpaid but it clearly carries an economic cost given that informal care time can usually be used for other purposes. These are all direct service costs. The indirect costs associated with time taken off work or school/college, or reduced productivity while at work, should also be measured for patients served by early intervention teams. By measuring such direct and indirect costs it is possible to see whether the extra costs associated with early intervention teams are offset by reduced costs elsewhere in the system, whether they are unchanged, or whether in fact they are increased as a result of these teams improving access to other forms of care.

\section{TYPES OF ECONOMIC EVALUATION}

A variety of methods are available for combining cost data with information on outcomes. The different types of analysis are distinguished according to the way in which outcomes are measured, and the choice between them depends crucially on the purpose of the evaluation.

\section{Cost-minimisation analysis}

A misconception about economic evaluation is that it is only concerned with the cost of different interventions. Although this is generally wrong, there may be situations when one is prepared to measure costs and to favour an intervention that costs less than an alternative. This would only be acceptable if it was known that the two interventions (for example early intervention and usual care) were equally effective. If that were the case, then the least costly would be the most efficient, other things being equal. Although economists will tend to warn against conducting such cost-minimisation analyses, decision-makers at local and national levels may be drawn towards them when resources are particularly tight.

\section{Cost-benefit analysis}

Like all forms of economic evaluation, cost-benefit analysis measures costs in monetary units, but it measures outcomes using monetary units also. In principle this makes cost-benefit analysis particularly powerful. If the monetised measure of outcome exceeds the costs, then the 
intervention produces a 'surplus', and when comparing two or more interventions, the one with the greatest surplus should be favoured. Comparisons with interventions in other sectors can be made if outcomes of these can also be measured using monetary units. However, the challenge with this method is that it is difficult to express mental health outcomes in monetary units, and studies that have done so have tended to focus on the economic value of gains in employment rather than clinical outcomes, for example reduced symptoms or improved functioning. It is possible to value such outcomes in monetary units using methods such as 'willingness to pay' but these have seldom been applied in mental health research.

\section{Cost-effectiveness analysis}

This form of evaluation may be of special relevance if the key question is how to provide appropriate care for a particular patient group, such as those with firstepisode psychosis. Cost-effectiveness analysis requires that a single outcome measure be chosen and this will usually be condition-specific. For example, in an evaluation of early intervention it may be appropriate to use a measure of functioning or symptomatology, or the DUP. When comparing early intervention with an existing alternative like standard care, costs will be combined with the outcome measure so that the intervention that produces the greatest outcome improvement for every pound spent can be identified. Although costeffectiveness is commonly used, it is not ideal for decision-makers, including commissioners, who have to decide how to spend healthcare funds across many different areas.

\section{Cost-consequences analysis}

Mental health problems affect people in numerous ways and therefore it may be inappropriate to focus entirely on one outcome measure as described above. Costconsequences analysis does not attempt to formally combine cost data with information on outcomes but presents cost and outcomes alongside each other to allow decision-makers to come to an overall conclusion regarding the different interventions being compared. Many evaluations will conduct a cost-consequences analysis to supplement a more rigorous cost-effectiveness analysis.

\section{Cost-utility analysis}

This is the form of analysis that is favoured by the National Institute for Health and Clinical Excellence (NICE) in the UK and by similar bodies in other countries. Costutility analysis uses a generic measure of outcome such that interventions across all areas of healthcare can, in principle, be compared. In the vast majority of costutility analyses the outcome measure is the quality-adjusted life year (QALY), where the time spent in a particular health state is adjusted according to the health-related quality of life (which is a proxy for utility) experienced during that time. Healthrelated quality of life is measured on a scale anchored by 1 (full health) and 0 (death). Therefore, if someone spends two years in a health state and during that time their quality of life is rated as 0.7 , they will have gained 1.4 QALYs (two times 0.7). Clearly, the challenge of this approach is to measure health-related quality of life in a meaningful way. One option is to use a simple rating scale, but more sophisticated methods are available such as defining health states according to the EuroQoL EQ-5D (Williams, 1995) or the Short Form 36-item questionnaire SF-36 (Ware et al, 1993) and then converting these into utility values.

\section{INTERPRETATION OF EVIDENCE FROM ECONOMIC EVALUATIONS}

The two most appropriate methods for evaluating early intervention services appear to be cost-effectiveness analysis and cost-utility analysis. If an early intervention service is compared with usual care using either of these approaches, then a number of results could occur, for example it would be appropriate to adopt an early intervention service if it results in lower costs than existing care and better outcomes. The early intervention service should also be favoured if outcomes are no different but costs are reduced or if costs are the same and outcomes are improved. Usual care would be the preferred option if the results were the opposite way round. However, it is unclear whether or not early intervention should be adopted if outcomes are better but costs are higher. In effect this becomes a value judgement that has to be made by decision-makers, with the key question being whether or not the increased costs are justified by the level of improved outcomes. It can also be seen that there is ambiguity about the appropriateness of an intervention if it saves money but is less effective. However, it is unlikely that this would apply to early intervention services as it is difficult to see how delaying treatment would produce better outcomes.

When the costs are higher and outcomes better, economists have tended to use incremental cost-effectiveness ratios (the difference in cost divided by the difference in outcomes) to show how much it costs for an intervention to produce an extra unit of outcome. More recently costeffectiveness acceptability curves have been used to indicate how much an extra unit of outcome (such as a point change on a symptom scale) would need to be valued in order for a particular intervention to be more cost-effective (or have greater costutility) than a comparator (e.g. McCrone et al, 2004).

\section{EVIDENCE PROVIDED FROM MODELLING}

Clinical evidence is usually generated from trials. There is a widely held view that trials should be randomised to reduce bias, but in evaluations of early intervention services this has not always proved possible and a number of observational studies have been carried out as well as randomised trials. The need for economic data can present further challenges: (a) trials may not be carried out over long enough periods to produce robust service use information; (b) evaluations may be pilot studies or have small samples; and (c) insufficient resources may be allocated for conducting an economic evaluation. In cases such as these an alternative approach is to use decision modelling, where the different consequences following a decision to adopt one intervention rather than another are mapped out. Costs and outcomes can then be attached to these different consequences or pathways. Decision modelling requires the availability of data with which to 'populate' the model. These data include probabilities (which indicate the likelihood of each consequence occurring), costs and outcomes, and can be obtained from a variety of sources such as existing randomised controlled trials, observational studies, routinely collected administrative data and expert opinion. Clearly there will be uncertainty around the appropriate value given to parameters and it is common to use sensitivity analyses in these circumstances to 
determine the extent to which the results of the model are affected by changes in these values.

\section{ECONOMIC EVIDENCE ON EARLY INTERVENTION SERVICES}

There is a growing body of evidence relating to the effectiveness of early intervention services. Some studies have evaluated (or are evaluating) interventions designed to prevent psychosis in people showing prodromal signs and symptoms, while other evaluations are of services provided to people in the early phases of actual psychosis. However, to date there have been very few studies which have provided information on the cost-effectiveness of early intervention services.

In Melbourne, Mihalopoulos et al (1999) compared the community-orientated treatment delivered by the Early Psychosis Prevention and Intervention Centre with standard care. A before-and-after study compared 51 patients treated in 1993 and 1994 with 51 matched retrospective controls receiving the pre-treatment model between 1989 and 1992. Outcomes assessed included quality of life and negative symptoms. Cost measures were limited to health services: in-patient stays, out-patient appointments, medication, community mental health team (CMHT) contacts, general practitioner (GP) contacts, private therapy and psychiatrist contacts. The Early Psychosis Prevention and Intervention Centre treatment was found to cost less than the pre-treatment model, although there was no indication of the statistical significance of this result. The cost saving arose because reductions in in-patient service use outweighed increases in community services. The study has a number of methodological limitations but encourages the view that an early intervention service can be more cost-effective than standard care.

In a large Danish randomised controlled trial (OPUS) enhanced assertive community treatment was compared to standard care for patients with first-episode schizophrenia (Petersen et al, 2005). Assertive community treatment resulted in significantly reduced psychotic symptoms, less substance misuse and greater satisfaction than standard care. Although an economic evaluation was not conducted it was shown that patients receiving assertive community

PAUL McCRONE, PhD, King's College London, Centre for the Economics of Mental Health, Health Services Research Department, Institute of Psychiatry, London, UK; MARTIN KNAPP, PhD, Personal Social Services Research Unit, London School of Economics and Political Science, London, UK

Correspondence: Paul McCrone, PO24 HSRD, Institute of Psychiatry, De Crespigny Park, London SE5 8AF, UK. Email: p.mccrone@iop.kcl.ac.uk

treatment had significantly fewer days in hospital during a 1-year follow-up period, although the difference after 2 years was not significant.

\section{ONGOING ECONOMIC EVALUATIONS}

A large evaluation, the EDEN study, of the implementation and effectiveness and costeffectiveness of early intervention services in the West Midlands region of the UK is currently being undertaken (see http:// www.pcpoh.bham.ac.uk/primarycare/ research/mental_health/Eden.htm. This will be a particularly informative study given the variety of urban and rural areas included.

In London, the Lambeth Early Onset (LEO) study (Craig et al, 2004) is evaluating the effectiveness of an early intervention service which is compliant with the 2001 policy implementation guide recommendations (Department of Health, 2001). A team delivering specialised care for patients with early psychosis has been found to be superior to standard care for maintaining contact with services, reducing readmissions to hospital, and improving social and vocational functioning, satisfaction and quality of life (Craig et al, 2004; Garety et al, 2006). An economic evaluation of the LEO service is being carried out. Service use data have been collected for patients receiving LEO or standard care and costs have been estimated. In order to assess cost-effectiveness the cost data are being combined with data on quality of life.

Another economic evaluation being conducted is of the Outreach and Support in South London (OASIS) prodromal service (Broome et al, 2005), also located in Lambeth. OASIS takes referrals from a variety of sources, but mainly GPs, the LEO service and from other adult and adolescent mental health services (Broome et al, 2005). To date there have not been any trials of the OASIS service and to assess its economic impact a decision model is being developed. This model will compare referral to OASIS with existing patterns of care. Key parameters in the model are the rates of transition to psychosis and the duration of untreated psychosis. Estimates for these parameters are being derived from local routine data and from information derived from the literature. The costs associated with a referral to OASIS or standard care are in the form of services used during the period of untreated psychosis, the impact on employment during that time and service contacts subsequent to the referral, such as formal and informal in-patient care and contacts with community services.

A further modelling exercise has been commissioned recently by the Department of Health. This will aim to assess the economic costs associated with early intervention schemes in general, i.e. early detection services as well as more conventional early intervention teams. Data to populate the model are being obtained from the various trials of early intervention services, and cost estimates are being made using data from the LEO study and routine data-sets.

\section{CONCLUSIONS}

There has been a rapid development of early intervention services in recent years. Healthcare resources are limited and, therefore, clinical and economic evaluations of early intervention services are required. However, while it is logical to assume that intervening in the early stage of any illness is beneficial, there is actually little evidence guiding the development of these services, and evidence from economic evaluations is particularly limited. A number of alternative ways of conducting economic evaluations are available, and cost-effectiveness analyses and cost-utility analysis appear especially useful.

\section{REFERENCES}

Broome, M. R., Wooley, J. B., Johns, L. C., et al (2005) Outreach and Support in South London (OASIS): implementation of a clinical service for prodromal 
psychosis and the at risk mental state. European Psychiatry, 20, 372-378.

Craig, T. K. J., Garety, P., Power, P., et al (2004) The Lambeth Early Onset (LEO) Team: randomised controlled trial of the effectiveness of specialised care for early psychosis. BM], 329, 1067 .

Department of Health (200I) The Mental Health Policy Implementation Guide. Department of Health

Garety, P. A., Craig, T. K. J., Dunn, G., et al (2006) Specialised care for early psychosis: symptoms, social functioning and patient satisfaction: randomised controlled trial. British Journal of Psychiatry, 188, 37-45.
McCrone, P., Knapp, M., Proudfoot, J., et al (2004) Cost-effectiveness of computerised cognitive behavioural therapy for anxiety and depression in primary care: randomised controlled trial. British Journal of Psychiatry, 185, 55-62.

Marshall, M., Lewis, S., Lockwood, A., et al (2006) Association between duration of untreated psychosis and outcome in cohorts of first-episode patients. Archives of General Psychiatry, 62, 975-983.

Mihalopoulos, C., McGorry, P. D. \& Carter, R. C. (1999) Is phase-specific community orientated treatment of early psychosis an economically viable method for improving outcome? Acta Psychiatrica Scandinavica, 100, 47-55.

Petersen, L., Jeppesen, P., Thorup, A., et al (2005) A randomised multicentre trial of integrated versus standard treatment for patients with a first episode of psychotic illness. BMI, 33I, 602-605.

Ware, J., Snow, K. K., Kosinski, M., et al (1993) SF-36 Health Survey, Manual and Interpretation Guide. The Health Institute, New England Medical Centre.

Williams, A. (1995) The Role of the EuroQoL Instrument in QALY Calculations. Centre for Health Economics, University of York. 